Draft VERSion July 26, 2021

Typeset using LATEX twocolumn style in AASTeX61

\title{
CHARACTERIZING ACCRETING DOUBLE WHITE DWARF BINARIES WITH THE LASER INTERFEROMETER SPACE ANTENNA AND GAIA
}

\author{
Katelyn Breivik, ${ }^{1,2}$ Kyle Kremer, ${ }^{1,2}$ Michael Bueno, ${ }^{3}$ Shane L. Larson, ${ }^{1,2}$ Scott Coughlin, ${ }^{2,4}$ And \\ VASSILIKI KALOGERA ${ }^{1,2}$
}

\footnotetext{
${ }^{1}$ Department of Physics 83 Astronomy, Northwestern University, Evanston, IL 60202, USA

${ }^{2}$ Center for Interdisciplinary Exploration 83 Research in Astrophysics (CIERA), Evanston, IL 60202, USA

${ }^{3}$ Department of Physics $\&$ Astronomy, Haverford College, Haverford, PA 19041

${ }^{4}$ School of Physics \& Astronomy, Cardiff University, The Parade, Cardiff, Wales, UK, CF24 $3 A A$
}

\begin{abstract}
We demonstrate a method to fully characterize mass-transferring double white dwarf (DWD) systems with a heliumrich (He) WD donor based on the mass-radius relationship for He WDs. Using a simulated Galactic population of DWDs, we show that donor and accretor masses can be inferred for up to $\sim 60$ systems observed by both LISA and Gaia. Half of these systems will have mass constraints $\Delta M_{\mathrm{D}} \lesssim 0.2 M_{\odot}$ and $\Delta M_{\mathrm{A}} \lesssim 2.3 M_{\odot}$. We also show how the orbital frequency evolution due to astrophysical processes and gravitational radiation can be decoupled from the total orbital frequency evolution for up to $\sim 50$ of these systems.
\end{abstract}

Keywords: white dwarfs — binaries — accretion — gravitational waves — astrometry — methods: numerical— methods: statistical 


\section{INTRODUCTION}

Double white dwarf (DWD) binaries, which make up the most substantial fraction of close binaries in the Milky Way (e.g., Marsh et al. 1995), will be a dominant source for future space-based interferometric gravitational-wave $(\mathrm{GW})$ detectors, such as the Laser Interferometer Space Antenna (LISA; Amaro-Seoane et al. (2013, 2017)). As gravitational radiation drives the components of a DWD binary together, it is possible for one of the stars to fill its Roche lobe, leading to the onset of mass-transfer.

The nature of mass-transferring DWDs has been explored both observationally (e.g., Warner and Woudt 2002; Strohmayer 2004a,b, 2005) and theoretically (e.g., Kremer et al. 2017; Gokhale et al. 2007; Marsh et al. 2004). Depending on the nature of the mass-transfer process, this can lead to the formation of an AM CVn system (e.g., Nather et al. 1981; Tutukov and Yungelson 1996; Nelemans et al. 2004) or a merger and Type Ia supernova (e.g., Maoz et al. 2014; Shen 2015). AM CVn systems in which both components are WDs provide astronomers with unique ways to use GW observations in combination with electromagnetic observations to probe the physics of mass-transfer and tidal processes.

Previous studies have shown that thousands of masstransferring DWDs are expected to be resolved by LISA (e.g., Nelemans et al. 2004; Ruiter et al. 2010; Kremer et al. 2017). Orbital evolution due to mass transfer or tides is expected to modify the orbital frequency evolution (chirp) from the pure gravitational radiation dominated chirp traditionally used in parameter estimation of observed systems (Valsecchi et al. 2012). Since LISA will observe the total orbital frequency evolution of an accreting DWD, an understanding of how astrophysically and gravitationally driven frequency evolution contribute to the total chirp is necessary to understand the system fully.

AM CVn systems with helium (He) WD donors that are undergoing stable mass-transfer are expected to follow well-constrained evolutionary tracks (e.g., Nelemans 2005; Deloye et al. 2007; Kalomeni et al. 2016). This is a consequence of the mass-radius relation for He-WDs and the way the orbits of these binaries respond to mass transfer.

In the coming years, Gaia is expected to provide distance and radial velocity measurements for more than a billion stars in the Milky Way, including a substantial population of DWD systems (Carrasco et al. 2014). Many of these systems are also expected to be observed by LISA, including several thousand detached (Korol et al. 2017) or mass-transferring systems (Kremer et al. 2017). In this Letter, we explore ways that measurements made by Gaia and LISA can be used in conjunction with the well-constrained evolutionary tracks expected for accreting DWDs with He-WD donors to place constraints upon various orbital parameters of these sys-

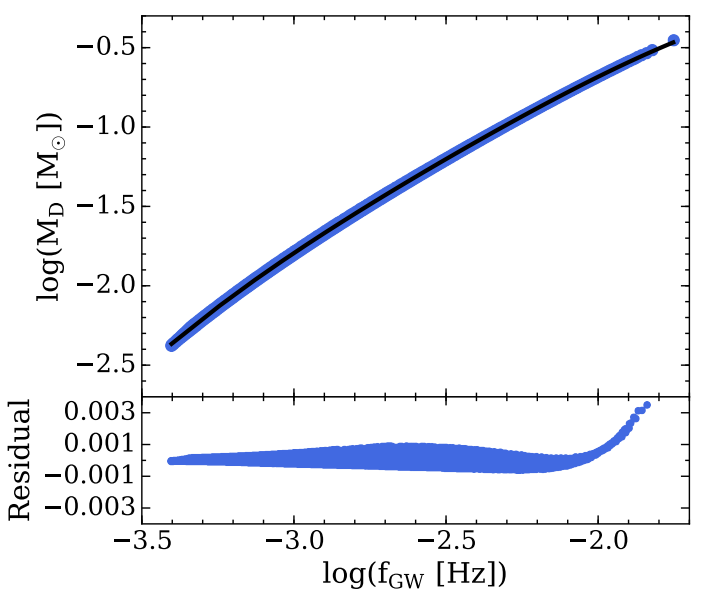

Figure 1. Donor mass vs GW frequency tracks for all modeled DWD systems and the residuals $\left(M_{\mathrm{D}}-M_{\mathrm{D}, \mathrm{fit}}\right)$ of the fit evaluated for each point on the tracks.

tems, including the component masses. Furthermore, such observations can be used to decouple the various components of the time-derivative of the orbital frequency for these systems.

In Section 2, we discuss well-determined evolutionary tracks for accreting DWD binaries with He-WD donors produced in our models. We discuss how these evolutionary tracks can be used to constrain the donor mass and mass-transfer rate for a particular DWD system, given an observation of the GW frequency for that system. In Section 3 we explore what can be learned from DWD systems that are observed by both Gaia and LISA. We conclude in Section 4.

\section{MEASUREMENTS OF MASS TRANSFER RATES AND DONOR MASSES}

Figure 1 shows the donor mass, $M_{\mathrm{D}}$, versus $\mathrm{GW}$ frequency, $f_{\mathrm{GW}}$, for the evolutionary tracks generated using the grid of initial donor and accretor masses modeled in Kremer et al. (2017). As in previous analyses (e.g., Marsh et al. 2004; Gokhale et al. 2007; Kremer et al. 2017), we assume all mass-transferring DWDs will be circular throughout their evolution. For circular binaries, $f_{\mathrm{GW}}=2 / P_{\mathrm{orb}}$, where $P_{\text {orb }}$ is the orbital period for the binary. The tracks shown here display the evolution of the binaries for all evolutionary time steps in which the time-derivative of the GW frequency (chirp) is negative (in the terminology of Kremer et al. (2017), $\dot{f}_{\text {tot }}<0$ ) from the onset of mass-transfer to $10 \mathrm{Gyr}$ through phases of both direct-impact and disk accretion. Each He-donor DWD that begins mass transfer will start at some point on this plot and evolve toward lower donor mass and lower GW frequency.

Recall from Kremer et al. (2017) that the initial semimajor axis for each of these systems is chosen such that the volume equivalent Roche-lobe radius of the donor is 
equal to the initial donor radius, as determined from the zero-temperature mass-radius $(\mathrm{M}-\mathrm{R})$ relation of Verbunt and Rappaport (1988).

As Figure 1 shows, $M_{\mathrm{D}}$ for all of modeled systems follow precise evolutionary tracks, independent of the initial system parameters. This narrow constraint is determined by the He-WD donor M-R relation. Similar evolutionary tracks have been shown before for accreting systems with He-WD donors (e.g., Nelemans 2005; Deloye et al. 2007; Kalomeni et al. 2016).

The relation demonstrated in Figure 1 shows that the mass-transfer rate and donor mass can be determined for mass-transferring DWDs if the GW frequency is known and the system is observed with a measurably negative chirp, indicating a mass-transferring system. We can represent this relationship by an analytic fit to a 4 th order polynomial using our simulated data:

$$
y=a+b x+c x^{2}+d x^{3}+e x^{4}
$$

where $x=\log \left(f_{\mathrm{GW}} / \mathrm{Hz}\right), y=\log \left(M_{\mathrm{D}} / M_{\odot}\right), a=$ $-2.1201, b=-4.2387, c=-3.0016, d=-0.7790$, and $e=-0.0791$. We choose a high order polynomial in order to closely follow the evolutionary track as well as provide a tractable method to infer donor mass for large numbers of observed systems.

We emphasize that the overall behavior of these evolutionary tracks is not unique to our mass-transfer models. As has been shown in several earlier analyses (e.g., Nelemans 2005; Deloye et al. 2007; Kalomeni et al. 2016), stable mass-transfer from a He-donor WD is expected to follow similar behavior regardless of the method used to model mass-transfer. Our analysis is most similar to Nelemans (2005). Deloye et al. (2007) use constant entropy $\mathrm{M}-\mathrm{R}$ relations found in Deloye \& Bildsten (2003) and Kalomeni et al. (2016) use Eq. 3 of Nelson \& Rappaport (2003) to determine the radius as a function of mass and chemical composition. We note that systems with exceptionally precise measurements due to close proximity in the Galaxy may be used to test the M-R relation for He-WDs. Since the orbital period, donor mass and donor radius are uniquely determined by the Roche lobe, systems where the donor mass can be independently verified provide a method to infer the donor radius.

\section{FULLY PARAMETERIZING THE SYSTEM WITH LISA AND GAIA}

The donor mass for any accreting DWD can be constrained if the system is observed to be transferring mass by LISA. If the same system is also observed by Gaia, the chirp mass can also be constrained, allowing the accretor mass to be calculated and the orbital chirp to be decoupled into its different components. We use the method described in Kremer et al. (2017) to model the evolution of accreting DWD binaries with He-WD donors and build a realistic Galactic population of Hedonor DWDs in the disk and bulge at the present day.

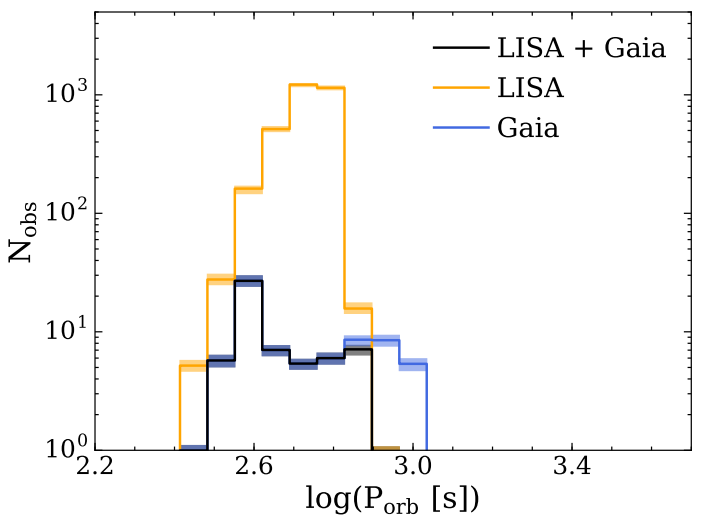

Figure 2. The number of systems observed as a function of orbital period in seconds. Systems observed with negative chirps by LISA are shown in orange. Systems with Gaia distance measurements are shown in blue. Systems observable in both cases are shown in black. The lines show the mode of our 100 population realizations and the spread shows spread $1 \sigma$ above and below this mode.

In addition to the models used in Kremer et al. (2017), we include five new models based on the results of Zorotovic et al. (2010); Toonen \& Nelemans (2013); Camacho et al. (2014) which suggest that the common envelope efficiency for WD-main sequence systems should be low $(\alpha \lesssim 0.2)$. We also include models which compute the binding energy of the stellar envelope $(\lambda)$ based on the stellar parameters and stellar type according to $\mathrm{Xu} \&$ Li (2010). Each of our models is listed in Table 1. We take $\alpha=0.25, \lambda=$ Var to be our fiducial model.

Our methods used to estimate the Gaia-detectability, including optical emission and extinction, are described in detail in the Appendix. We note that the methods demonstrated can be applied to any simulated data sets with He-WD donors due to the expected behavior from the $\mathrm{M}-\mathrm{R}$ relationship for He-WDs.

We generate 100 population realizations for the Milky Way disk and bulge using the Compact Object Synthesis and Monte-Carlo Code (COSMIC) according to the methods described in Kremer et al. (2017). We use these populations to investigate the overall number and binary parameters of accreting He-donor DWDs observable by both LISA and Gaia. Figure 2 shows the number and orbital period distribution of He-donor DWDs observed with negative chirps by LISA (green) and with measured distances by Gaia (blue) as well as systems observed by both missions (black) as a function of orbital period. From our 100 population realizations for the fiducial model, we find, on average, $\sim 3000$ systems resolved with negative chirps by $L I S A, \sim 80$ systems observed by Gaia, and $\sim 60$ systems with resolved negative chirps and measured distances. The numbers for each of the common envelope models in Kremer et al. (2017) as well as our newly run models are summarized 
Table 1. Average number of He-donor DWDs observed with negative chirps by LISA $\left(N_{L I S A}\right)$, measured distances by Gaia $\left(N_{\text {Gaia }}\right)$, and systems satisfying both conditions ( $\left.N_{\text {both }}\right)$. The fiducial model is $\alpha=0.25, \lambda=$ Var.

\begin{tabular}{cccc}
\hline Model & $N_{\text {LISA }}$ & $N_{\text {Gaia }}$ & $N_{\text {both }}$ \\
\hline$\alpha=0.25, \lambda=\operatorname{Var}$ & 3077 & 78 & 61 \\
$\alpha=0.25, \lambda=1.0$ & 3410 & 114 & 94 \\
\hline$\alpha=0.5, \lambda=\operatorname{Var}$ & 3917 & 116 & 95 \\
$\alpha=0.5, \lambda=1.0$ & 2757 & 73 & 60 \\
\hline$\alpha=1.0, \lambda=\operatorname{Var}$ & 8295 & 84 & 9 \\
$\alpha=1.0, \lambda=0.1$ & 77 & 209 & 8 \\
$\alpha=1.0, \lambda=1.0$ & 6225 & 2305 & 518 \\
$\alpha=1.0, \lambda=10.0$ & 3684 & 1488 & 256 \\
\hline
\end{tabular}

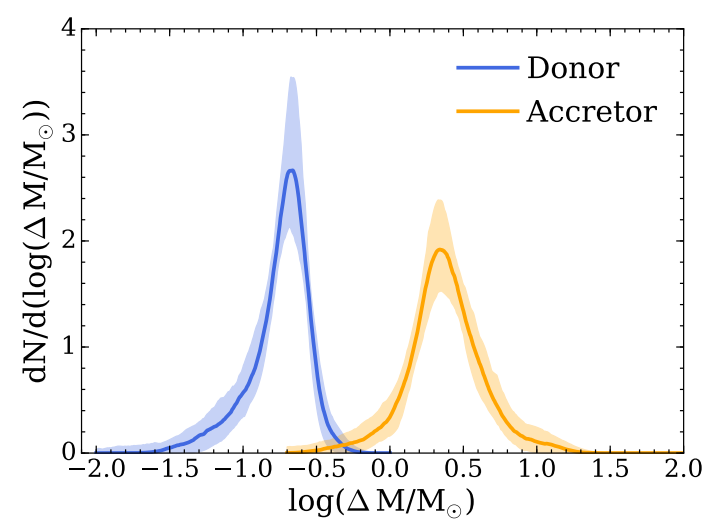

Figure 3. Measurement errors for donor masses (blue) and accretor masses (orange). The shaded regions show $5-95 \%$ percentile spread for our 100 population realizations.

in Table 1. Broadly, increasing the common envelope efficiency leads to higher numbers of observed systems.

\subsection{Decoupling the component masses}

For circular binaries, the signal-to-noise ratio $(\mathrm{S} / \mathrm{N})$ is given by

$$
\mathrm{S} / \mathrm{N} \approx \frac{\mathrm{h}_{0} \sqrt{\mathrm{T}_{\mathrm{obs}}}}{\mathrm{h}_{\mathrm{f}}},
$$

where $h_{0}$ is the scaling amplitude,

$$
h_{0}=4 \frac{G}{c^{2}} \frac{M_{c}}{D}\left(\frac{G}{c^{3}} \pi f_{\mathrm{GW}} M_{c}\right)^{2 / 3},
$$

$T_{\text {obs }}$ is the observational time of the LISA mission, taken here, as in Kremer et al. (2017) to be 4 years, $h_{\mathrm{f}}$ is the spectral amplitude value for a specified gravitationalwave frequency, $f_{\mathrm{GW}}$, given by the standard $L I S A$ sen- sitivity curve in Larson et al. (2002), and $M_{c}$ is the chirp mass defined as

$$
M_{c}=\frac{\left(M_{\mathrm{A}} M_{\mathrm{D}}\right)^{3 / 5}}{\left(M_{\mathrm{A}}+M_{\mathrm{D}}\right)^{1 / 5}} .
$$

A parallax distance measurement, $D$, obtained from Gaia can be combined with the LISA observation of $h_{0}$ and $f_{\mathrm{GW}}$ to directly compute $M_{c}$ from equation 3 . Using the donor mass determined with the method of Section 2, the accretor mass can be constrained.

We assume LISA measurement errors using Eqs. 1214 of Takahashi \& Seto (2002), which are valid for our data set containing GW frequencies $10^{-4} \mathrm{~Hz}<f_{G W}<$ $10^{-2} \mathrm{~Hz}$. We list them here for convenience:

$$
\begin{aligned}
\frac{\Delta h_{0}}{h_{0}} & =0.2\left(\frac{S / N}{10}\right)^{-1} \\
\Delta f_{\mathrm{GW}} & =0.22\left(\frac{S / N}{10}\right)^{-1}\left(\frac{T_{\mathrm{obs}}}{\mathrm{yr}}\right)^{-1} \\
\Delta \dot{f}_{\mathrm{tot}} & =0.43\left(\frac{S / N}{10}\right)^{-1}\left(\frac{T_{\mathrm{obs}}}{\mathrm{yr}}\right)^{-2} .
\end{aligned}
$$

We assume the Gaia distance measurement error to be

$$
\frac{\Delta D}{D}=\frac{\Delta \alpha}{\alpha}
$$

where the distance is in pc and $\alpha$ is the Gaia magnitudedependent astrometric accuracy in arcsec taken from Gaia Collaboration et al. (2016b). These measurement errors can be propagated through our equations for $h_{0}$, $M_{c}$ and $M_{\mathrm{D}}$ to obtain measurement errors for both component masses.

We note that systems with parallax measurement errors in excess of $20 \%$ (approximately half of our resolved systems) will not follow this simple relation (BailerJones 2015). For the purposes of this initial study, we use the approximation of Eq. 9 and leave a more detailed treatment of distance errors for a later study.

Figure 3 shows the distribution of our mass measurement errors for both $M_{D}$ and $M_{A}$ as well as the percent error between the mean 'inferred' masses from observations and our $M_{\mathrm{D}}-f_{\mathrm{GW}}$ fit and the 'true' simulated values. The peak in the $\Delta M_{\mathrm{D}}$ distribution is $\sim 0.2 M_{\odot}$ while the peak for the $\Delta M_{\mathrm{A}}$ distribution is $\sim 2 M_{\odot}$. The 50th percentile of our data $(\sim 30$ systems $)$ have mass measurements better than $\Delta M_{\mathrm{D}} \simeq 0.2 M_{\odot}$ and $\Delta M_{\mathrm{A}} \simeq 2.3 M_{\odot}$.

\subsection{Decoupling the astrophysical chirp from the gravitation-radiation chirp}

As in Kremer et al. (2017), the chirp for masstransferring DWDs can be broken into three separate components

$$
\dot{f}_{\text {total }}=\dot{f}_{\mathrm{GR}}+\dot{f}_{\mathrm{MT}}+\dot{f}_{\text {tides }}
$$




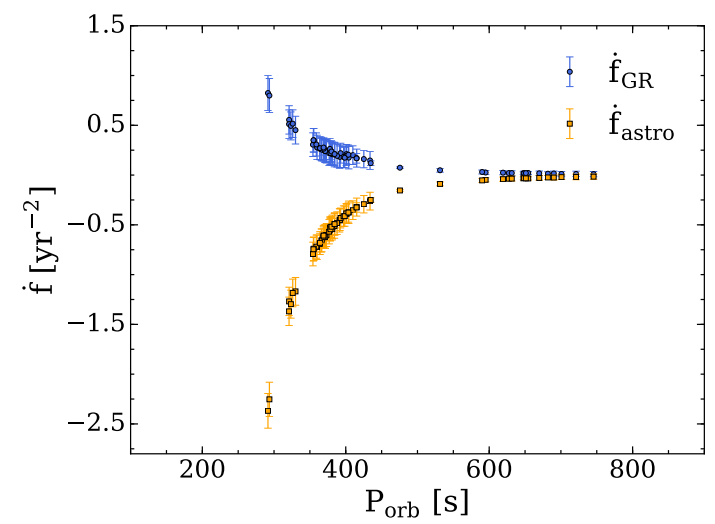

Figure 4. GR (blue) and astrophysical (orange) chirps as a function of GW frequency. The Error bars show the $1 \sigma$ measurement errors. See Eqs. 10-12 for an explanation of the difference between $\dot{f}_{\mathrm{GR}}$ and $\dot{f}_{\text {astro }}$.

where $\dot{f}_{\mathrm{GR}}, \dot{f}_{\mathrm{MT}}$, and $\dot{f}_{\text {tides }}$ are the contributions to the chirp due to gravitational radiation, mass transfer, and tidal interactions, respectively. It is convenient to group $\dot{f}_{\mathrm{MT}}$ and $\dot{f}_{\text {tides }}$ into a single quantity defined as the astrophysical chirp, $\dot{f}_{\text {astro }}$, so that $\dot{f}_{\text {total }}=\dot{f}_{\text {GR }}+\dot{f}_{\text {astro }}$.

Here $\dot{f}_{\mathrm{GR}}$ is given by

$$
\dot{f}_{\mathrm{GR}}=\frac{96}{5} \frac{f_{\mathrm{GW}}^{2}}{\pi c^{5}}\left(G \pi f_{\mathrm{GW}} M_{c}\right)^{5 / 3} .
$$

If $M_{c}$ is computed using $D$ obtained from a Gaia observation, as described in Section 3.1, $\dot{f}_{\mathrm{GR}}$ can be computed directly. If, additionally, the system has a sufficiently high $\dot{f}_{\text {total }}$ to be measured directly by LISA (for $T_{\text {obs }}=4$ years, $\dot{f}_{\min }=7.93 \times 10^{-10} \mathrm{~Hz} \mathrm{yr}^{-1}$; see Kremer et al. (2017) for further detail), the observed $\dot{f}_{\text {total }}$ and calculated $\dot{f}_{\mathrm{GR}}$ values can be used to solve for the astrophysical contribution to the total chirp

$$
\dot{f}_{\text {astro }}=\dot{f}_{\text {total }}-\dot{f}_{\text {GR }} \text {. }
$$

We propagate the measurement errors $\Delta h_{0}, \Delta f_{\mathrm{GW}}$, $\Delta \dot{f}_{\text {total }}$, and $\Delta D$ through Eqs. 11 and 12 to obtain the inferred values of $\dot{f}_{\mathrm{GW}}$ and $\dot{f}_{\text {astro }}$. Figure 4 shows $\dot{f}_{\mathrm{GR}}$ (blue) and $\dot{f}_{\text {astro }}$ (green) and their $1 \sigma$ measurement error for the LISA-resolved systems taken from a single sample realization for our fiducial model. On average, we find $\sim 50$ systems with $P_{\text {orb }} \lesssim 800 s$ have resolvable gravitational radiation and astrophysically driven chirps from our Galactic realizations.

\section{CONCLUSION}

We have explored the evolution of accreting DWD binaries with He-WD donors and demonstrated these systems provide unique laboratories for probing the physics of mass-transfer in binary systems. We have also shown that if the GW frequency for these systems is obtained using LISA, the donor mass can be constrained using well-determined evolutionary tracks. The method demonstrated above presents a previously undescribed analysis for inferring donor and accretor masses using multi-messenger observations of accreting DWDs. We note that if the systems are eclipsing, the binary may be fully parameterized by solving the 'visual binary' problem. However, this requires a narrow range of orbital inclinations that will greatly reduce the overall number of characterized systems.

Furthermore, we have demonstrated that of the several thousand accreting DWDs observable by LISA, $\sim 680$ are expected to also be observed by Gaia. For systems observed by both LISA and Gaia, we have shown that in addition to the donor mass, the chirp mass, and therefore the accretor mass, can also be constrained to $\Delta M_{\mathrm{D}} \lesssim 0.2 M_{\odot}$ and $\Delta M_{\mathrm{A}} \lesssim 2.3 M_{\odot}$ accuracy for $\sim 30$ He-donor DWDs. Additionally, the chirp for $\sim 50$ systems can be decoupled into its GW and astrophysical components. These numbers vary if different binary evolution models are used (see Table 1), however the methods of inferring binary parameters are agnostic to these models. Thus, while the overall number of observed accreting He-donor DWDs may change, the ability to constrain the donor mass, accretor mass, and orbital frequency evolution remains.

The method demonstrated above relies on an understanding of the mass-radius relationship that governs He-WDs. While several models currently exist, future observations of mass-transferring DWDs with He-WD donors are needed to properly constrain the physics. Current and future observing campaigns like the Gaia mission and the Large Synoptic Survey Telescope (LSST Science Collaboration et al. 2009) show promise to discover such systems and, through follow-up observations, constrain the He-WD mass-radius relation before LISA's launch.

KB gratefully acknowledges Chris Pankow's helpful discussion. $\mathrm{KB}$ and $\mathrm{KK}$ also gratefully acknowledge Michael Zevin for helpful discussion. KK acknowledges support from the National Science Foundation Graduate Research Fellowship Program under Grant No. DGE1324585. KB and SLL acknowledge support from NASA Grant NNX13AM10G. VK acknowledges support from Northwestern University. The majority of our analysis was performed using the computational resources of the Quest high performance computing facility at Northwestern University which is jointly supported by the Office of the Provost, the Office for Research, and Northwestern University Information Technology. 
Amaro-Seoane, P. et al. 2013, arXiv: 1305.5720

Amaro-Seoane, P. et al. 2017, arXiv: 1702.00786

Bailer-Jones, C. A. L. 2015, PASP, 127, 994

Camacho, J., Torres, S., García-Berro, E., et al. 2014, A\&A, 566, A86

Cardelli, J. A., Clayton, G. C., \& Mathis, J. S. 1989, ApJ, 345,245

Carrasco, J. M., Catalán, S., Jordi, C., et al. 2014, A\&A, $565, \mathrm{~A} 11$

Deloye, C. J., \& Bildsten, L. 2003, ApJ, 598, 1217

Deloye, C. J., Taam, R. E., Winisdoerffer, C., \& Chabrier, G. MNRAS, 381, 525

Eggleton, P. P. 1983, ApJ, 268, 368.

Flower, P. J. 1996, ApJ, 469, 355

Gaia Collaboration, Brown, A. G. A., Vallenari, A., et al. 2016, A\&A, 595, A2

Gokhale, V., Peng, X. M. and Frank, J. 2007, ApJ 655, 1010.

Hansen, B. M. S. 1999, ApJ, 520, 680

Hurley, J. R., Tout, C. A., \& Pols, O. R. 2002, MNRAS, $329,897$.

Jordi, C., Gebran, M., Carrasco, J. M., et al. 2010, A\&A, 523, A48

Kalomeni, B., Nelson, L., Rappaport, S. et al. 2016, ApJ, 833,83

Korol, V., Rossi, E. M., Groot, P. J., et al. 2017, MNRAS, 470, 1894

Kremer, K., Breivik, K., Larson, S. L., \& Kalogera, V. 2017, ApJ, 846, 95

Larson, S. L., Wellings, R. W., \&Hiscock, W. A., PhRvD, 66, 062001

LSST Science Collaboration, Abell, P. A., Allison, J., et al. 2009, arXiv:0912.0201

Maoz, D., Mannucci, F., and Nelemans, G. 2014, AARA, $52,107$.

Marsh, T. R., Dhillon, V. S. and Duck, S. R. 1995, MNRAS, 275, 828.

\section{REFERENCES}

Marsh, T. R., Nelemans, G. and Steeghs, D. 2004, MNRAS, 350, 113.

Nather, R. E., Robinson, E. L. and Stover, R. J. 1981, ApJ, $244,269$.

Nelemans, G., Portegies Zwart, S. F., Verbunt, F., \& Yungelson, L. R. 2001, A\&A, 368, 939

Nelemans, G., Yungelson, L. R., \& Portegies Zwart, S. F. 2004, MNRAS, 349, 181

Nelemans, G. The Astrophysics of Cataclysmic Variables and Related Objects, Proceedings of ASP Conference Vol. 330. Edited by J.-M. Hameury and J.-P. Lasota. San Francisco: Astronomical Society of the Pacific, 2005., p.27

Nelson, L. A., \& Rappaport, S. 2003, ApJ, 598, 431

Pringle, J. E. 1977, MNRAS, 178, 195

Pringle, J. E. 1981, ARA\&A, 19, 137

Ruiter, A. J., Belczynski, K., Benacquista, M., Larson, S. L., \& Williams, G. 2010, ApJ, 717, 1006

Sandage, A. 1972, ApJ, 178, 1

Shen, K. 2015, ApJL,805, L6.

Strohmayer, T. 2004a, ApJ 610, 416.

Strohmayer, T. 2004b, ApJ 614, 358.

Strohmayer, T. 2005, ApJ, 627, 920

Takahashi, R., \& Seto, N. 2002, ApJ, 575, 1030

Torres, G. 2010, AJ, 140, 1158

Toonen, S., \& Nelemans, G. 2013, A\&A, 557, A87

Tutukov, A. and Yungelson, L. 1996, MNRAS, 280, 1035.

Valsecchi, F., Farr, W. M., Willems, B., Deloye, C. J., \& Kalogera, V. 2012, ApJ, 745, 137

Verbunt, F. and Rappaport, S. 1988, ApJ, 332, 193

Wade, R. A. 1984, MNRAS, 208, 381

Warner, B. and Woudt, P. 2002, PASP 792, 129

Xu, X.-J., \& Li, X.-D. 2010, ApJ, 716, 114

Zorotovic, M., Schreiber, M. R., Gänsicke, B. T., \& Nebot Gómez-Morán, A. 2010, A\&A, 520, A86 


\section{APPENDIX}

\section{A. MODELING THE OPTICAL EMISSION}

Here we introduce our method for modeling the electromagnetic emission of accreting DWD systems. We explore optical emission from the component stars and the disk itself.

\section{A.1. Emission from WD components}

As in Nelemans et al. (2004), we consider three sources of optical emission: (1) the donor, (2) the accretor, and (3) the accretion disk (if present). The optical emission from the donor and accretor is modeled as the cooling luminosity of the WD. We use the cooling functions of Nelemans et al. (2004), which are approximations to those of Hansen (1999):

$$
\log L=L_{\max }-1.33 \log \left(\frac{t}{10^{6} \mathrm{yrs}}\right)
$$

where $L_{\max }$ is given by:

$$
L_{\max }= \begin{cases}1-\left(0.9-M_{\mathrm{WD}}\right), & M_{\mathrm{WD}} \geq 0.5 M_{\odot} \\ 1.4-1.33\left(0.45-M_{\mathrm{WD}}\right), & M_{\mathrm{WD}}<0.5 M_{\odot}\end{cases}
$$

In this simple analysis, as in Nelemans et al. (2004), we do not consider heating of the accretor due to accretion.

Assuming, the WDs radiate as blackbodies, the temperature of each star is given by $L=4 \pi R_{\mathrm{WD}}^{2} \sigma T^{4}$. From the temperature and appropriate bolometric correction, the optical emission from each component can be calculated.

\section{A.2. Emission from disk}

The accretion luminosity for a mass-transferring binary is given by

$$
L_{\mathrm{acc}}=G M_{A} \dot{M}\left(\frac{1}{R_{A}}-\frac{1}{R_{\mathrm{L} 1}}\right)
$$

If an accretion disc is present, we assume half of the accretion luminosity is radiated by the disk itself giving

$$
L_{\mathrm{disk}}=\frac{1}{2} G M_{A} \dot{M}\left(\frac{1}{R_{A}}-\frac{1}{R_{\mathrm{L} 1}}\right),
$$

with the other half being radiated at the boundary layer. Here, $R_{A}$ is the accretor radius, $\dot{M}$ is the mass-transfer rate, and $R_{\mathrm{L} 1}$ is the distance of the first Lagrangian point to the center of the accretor. For circular binaries, $R_{\mathrm{L} 1}=a-R_{\mathrm{L}}$, where $R_{\mathrm{L}}$ is the Roche-lobe radius as determined by Eggleton (1983).

We assume the disk has a radial temperature profile,(Pringle 1981)

$$
T(R)=\left(\frac{3 G M_{A} \dot{M}}{8 \pi r^{3} \sigma}\left[1-\left(R_{A} / R\right)^{1 / 2}\right]\right)^{1 / 4},
$$

and is made up of 10 equally-radially-spaced annuli, each radiating as a blackbody. As in Nelemans et al. (2001), we take the outer radius of the disk to be $R_{\text {out }}=0.7 R_{R_{\mathrm{L} 1}}$. We compute visual magnitudes, $m_{V}$, and $B-V$ colors from the bolometric corrections in Flower (1996) with corrections of Torres (2010) using the blackbody luminosity and effective temperature of the disk. The Gaia G-magnitude is then computed from the color-color transformations of Jordi et al. (2010).

For the sake of comparison, we also take interstellar reddening into account using the simple Sandage (1972) extinction model used in Nelemans et al. (2004):

$$
A_{V}(\infty)= \begin{cases}0.165 \frac{\left[\tan \left(50^{\circ}\right)-\tan (b)\right]}{\sin (b)} & \text { for } b<50^{\circ} \\ 0 & \text { for } b \geq 50^{\circ},\end{cases}
$$

where $b$ is the galactic latitude. To apply this extinction model to our DWD populations, we account for the extinction of each population by computing the integrated spatial distribution of each galactic component (disk, bulge) and multiply by the total extinction. For our disk population, we compute $A_{V}(d)_{d i s k}$ as

$$
A_{V}(d)_{\text {disk }}=A_{V}(\infty) \tanh \left(\frac{d \sin (b)}{z_{h}}\right),
$$


where $d$ is the distance to each DWD and $z_{h}=0.352 \mathrm{kpc}$. For the bulge, we compute $A_{V}(d)_{\text {bulge }}$ as

$$
A_{V}(d)_{\text {bulge }}=A_{V}(\infty) \operatorname{Erf}\left(\frac{d \sin (b)}{R_{h}}\right),
$$

where $R_{h}=0.5 \mathrm{kpc}$.

We use the color transformations in Cardelli et al. (1989) to convert the $V$-band extinction to $G$-band extinction using the center of the Gaia wavelength band $\lambda_{G}=673 \mathrm{~nm}$. Here we also note that the maximal extinction is $A_{V} \leq 0.2$ which gives $A_{\lambda_{G}} \leq 0.16$. Though our extinction model is simplified, we note that the overall effects from reddening on the number of DWDs detectable by Gaia are outweighed by the differences of our binary evolution models. Thus we do not expect that using a different extinction model will have a significant effect on our end results. 